\title{
Etude de quelques caractéristiques d'une variété fromagère traditionnelle iranienne appelée "Fromage en outre " ${ }^{(*)}(*)$
}

par

\author{
H. LAMÉ et M. HEKMATI \\ Maîtres de conférences au département des Industries Agricoles \\ de la Faculté d'Agronomie de l'Université de Téhéran \\ Karadj (Iran)
}

\section{INTRODUCTION}

Dans les régions de Tavalèche et Assalème, situées au sud de la mer Caspienne, on fabrique, à partir du lait de brebis ou un mélange de lait de brebis et de chèvre, une variété de fromage dont la technologie traditionnelle est transmise de génération en génération depuis des siècles. L'affinage et la conservation de ce fromage se réalisent dans des outres fabriquées à partir de la peau des moutons et des chèvres après leur abattage, d'où son nom.

Bien que la technologie utilisée soit plus ou moins rudimentaire et ne tienne pas compte de certaines règles hygiéniques et de progrès modernes, le produit fini est très savoureux et peut concurrencer de ce point de vue certains fromages étrangers.

L'étude des conditions de fabrication, de la qualité et de la propreté du lait et du matériel utilisés permettrait, sans doute, de prendre des mesures rationnelles afin d'instruire les fabricants pour une production meilleure.

Il est à rappeler que l'on fabrique en Iran deux variétés de fromage en outre. L'une est celle de Tavalèche et Assalème qui fait le sujet de cette étude, et l'autre est une variété qui ressemble, à certains points de vue technologiques, au fromage en pot [1] étudié auparavant. Ce dernier fera le sujet d'une prochaine publication.

Le but de ce travail est de connaître le procédé traditionnel de fabrication et certaines caractéristiques du fromage en outre de Tavalèche et Assalème tel qu'il est fabriqué de nos jours.

(*) Ce fromage s'appelle en iranien « Panir-é-khiki ».

(**) Le crédit de cette étude est fourni par le fonds de recherches de l'Université de Téhéran. 


\section{TECHNOLOGIE TRADITIONNELLE}

Pour fabriquer du fromage en outre on utilise, habituellement, du lait entier de brebis ou un mélange de lait de brebis et de chèvre trait à la main. Parfois, surtout en fin de lactation, du lait partiellement écrémé est aussi utilisé. La crème ainsi obtenue sert à la fabrication du beurre à usage domestique.

L'emprésurage du lait se fait la plupart du temps avec de la présure naturelle et peu après la traite, quand le lait est encore tiède, dans des bacs (marmites) en cuivre étamé. Quand l'écrémage partiel du lait est exigé, l'emprésurage se fait, naturellement, après cette opération.

Le lait se coagule $2 \mathrm{~h}$ environ après l'emprésurage. On procède alors à la cuisson du caillé dans son sérum avant de le séparer. La cuisson du caillé se réalise pendant une $1 / 2 \mathrm{~h}$ environ à feu doux (à $50^{\circ} \mathrm{C}-60^{\circ} \mathrm{C}$ environ) dans les mêmes bacs où l'emprésurage du lait est effectué.

L'égouttage et le pressage du caillé sont réalisés après la cuisson dans des vases en bois fabriqués à partir de troncs d'arbres forestiers et appelés "Tchanaghes $\left({ }^{*}\right)$ ». Il est bien évident que les dimentions des Tchanaghes dépendent de celles des arbres à partir desquels ils sont fabriqués. Cependant, les plus courants sont ceux qui ont un diamètre d'ouverture de $50 \mathrm{~cm}$ et de $30 \mathrm{~cm}$ de profondeur.

Le pressage dure environ $15 \mathrm{~h}$ à la température ambiante $\left(18^{\circ} \mathrm{C}\right.$ à $22^{\circ} \mathrm{C}$ environ) en déposant des poids sur le caillé contenu dans les Tchanaghes.

On procède alors au découpage du caillé pressé en morceaux pesant approximativement 0,5 à $1 \mathrm{~kg}$ qui seront immergés, par la suite, dans de la saumure à 10 p. 100 de sel environ contenue dans des outres.

Cinq jours plus tard la saumure est renouvelée. La nouvelle saumure a la même concentration en sel que la précédente.

Après avoir bien rempli les outres de fromage et les espaces vides de saumure, on ferme l'orifice des outres hermétiquement en utilisant des fibres végétales, comme la ficelle.

Les outres ainsi remplies sont placées ensuite dans des caves peu éclairées et fraîches à une température de $12^{\circ} \mathrm{C}$ à $15^{\circ} \mathrm{C}$ environ. moins.

On commence à consommer le fromage 1 mois plus tard au

(*) Le mot « Tchanaghe » est probablement emprunté à la langue turque et signifie « le bol ». 
La fabrication du fromage en outre s'effectue habituellement au printemps pendant la période naturelle de lactation des brebis et des chèvres, puis est consommé tout au long du reste de l'année.

Les Tchanaghes sont conservés, après l'usage, au frais à l'abri du soleil et de la chaleur du feu.

Le sel utilisé pour la préparation des saumures est du sel gemme provenant des mines de la région de Khalkhal (Azerbaïdjan).

\section{MATERIELS ET METHODES}

\section{1) Matériels}

Origine et transport des échantillons jusqu'au laboratoire.

$\mathrm{Au}$ cours des visites effectuées pendant 10 mois environ (du début juin jusqu'à fin mars) dans les villages Klour, Kapour-Tchal, Rézvandeh, Dorkhaneh, Ardehdjan, Dinal-Tchal, Assalème, HachtPar, Nassal et Khouchab-Sar, nous avons prélevé des échantillons dans des outres dont le contenu était en cours de vente ou consommation.

Les échantillons ainsi prélevés ont été transportés dans des bocaux stériles en matière plastique rangés dans des caisses frigorifiques.

Pour éviter toute perte d'humidité et les conséquences qui pourraient en résulter pour le produit, les échantillons ont été immergés dans une portion de saumure où ils étaient immergés avant le prélèvement.

\section{2) Méthodes}

$1^{\circ}$ Les échantillons sont homogénéisés séparément en évitant toute contamination extérieure. Puis, $1 \mathrm{~g}$ de chaque échantillon homogénéisé est broyé dans un mortier de porcelaine stérile, préalablement chauffé à $45^{\circ} \mathrm{C}$ en ajoutant petit à petit $1 \mathrm{ml}$ d'une solution de citrate de soude stérile à 20 p. 100 . On ajoute finalement $8 \mathrm{ml}$ d'eau stérile de $45^{\circ} \mathrm{C}$ tout en continuant à triturer.

$2^{\circ}$ La matière sèche totale est mesurée par la méthode décrite AOAC [2] à $105^{\circ} \mathrm{C}$.

3. Dosage de l'azote total (NT) et de l'azote soluble (NS) par la méthode de Kjeldahl décrite par Kasikowski [3].

$4^{\circ}$ La détermination de la teneur en matière grasse a été effectuée en utilisant la méthode de Gerber [4].

$5^{\circ}$ Les mesures de $\mathrm{pH}$ ont été effectuées par potentiométrie à l'aide d'un pHmètre de marque Fucoma (Knick) muni d'une électrode combinée. 
$6^{\circ}$ La détermination de la teneur en $\mathrm{NaCl}$ a été faite par la méthode décrite par Newlander et Atherton [5].

\section{RESULTATS ET CONCLUSIONS}

Comme il est montré dans le tableau 1, la teneur en matière sèche des échantillons analysés se situe entre 37,60 pour l'échantillon $n^{\circ} 5$ et 59 pour l'échantillon $n^{\circ} 4$.

La teneur en matière grasse (MG) va de 35,38 (échantillon $n^{\circ} 7$ ) à 52,05 (échantillon $n^{\circ}$ 6) p. 100 de la matière sèche, ou bien de 14,33 (échantillon $\mathrm{n}^{\circ}$ 5) à 29,83 (échantillon $\mathrm{n}^{\circ} 4$ ) p. $100 \mathrm{~g}$ de fromage.

TABLEAU 1

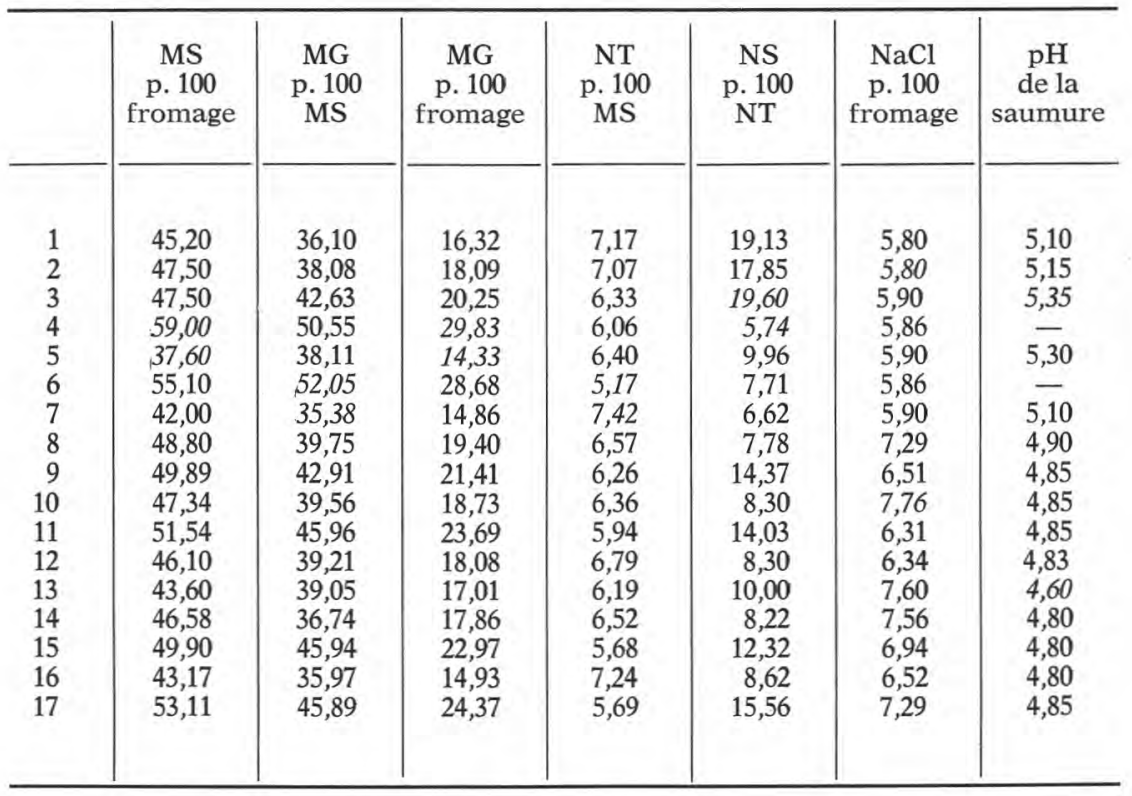

En ce qui concerne l'azote total (NT) en p. 100 de la matière sèche (MS), il va de 5,17 (échantillon $n^{\circ} 6$ ) à 7,42 (échantillon $n^{\circ} 7$ ).

L'azote solubilisé (NS) mesuré au cours de nos essais était toujours inférieur à $1 / 5$ de l'azote total et compris entre 5,74 (échantillon $n^{\circ} 4$ ) et 19,6 (échantillon $n^{\circ} 3$ ) p. 100 de l'azote total.

La quantité de sel absorbé varie également selon l'échantillon. Il est de 5,80 g pour l'échantillon $\mathrm{n}^{\circ} 2$ et de 7,79 g pour l'échantillon $\mathrm{n}^{\circ} 10$, p. $100 \mathrm{~g}$ de fromage, indiquant les deux valeurs extrêmes. 
Quant au $\mathrm{pH}$ des saumures où nous avons pris nos échantillons de fromage, il est compris entre 4,60 pour l'échantillon $n^{\circ} 13$ et 5,35 pour l'échantillon $\mathrm{n}^{\circ} 3$.

Les résultats exprimés plus haut et insérés dans le tableau 1 montrent une telle diversité que l'on ne peut pas dire qu'il s'agit d'une seule mais plutôt d'un ensemble de variétés ayant quelques points communs dans leurs procédés de fabrication.

Cette diversité de caractères ou plutôt de variétés résulte, à notre avis, des facteurs suivants :

1) Origine et différence de composition du lait utilisé.

2) Concentration en sel des saumures.

3) Température des locaux de conservation.

4) Durée de conservation.

Cependant les valeurs relatives à l'azote soluble, au moins pour la plupart des échantillons analysés, prouvent qu'il s'agit d'un fromage affiné.

Les dénombrements microbiens réalisés sur les 10 premiers échantillons montrent, pour la plupart des échantillons, une contamination par les bactéries coliformes (tab. 2). Cette contamination

TABLEAU 2

\begin{tabular}{c|c|c}
\hline & Coliformes $(\mathrm{n} / \mathrm{g})$ & Flore totale $(\mathrm{n} / \mathrm{g})$ \\
\hline & & \\
\hline 2 & 10 & $11 \times 10^{3}$ \\
3 & 9 & $10 \times 10^{3}$ \\
4 & 0 & $4 \times 10^{3}$ \\
5 & 2 & $4 \times 10^{3}$ \\
6 & 10 & $2 \times 10^{3}$ \\
7 & 70 & $13 \times 10^{3}$ \\
8 & 0 & $4 \times 10^{3}$ \\
9 & 90 & $6 \times 10^{3}$ \\
10 & 320 & $3 \times 10^{3}$ \\
& & $20 \times 10^{3}$ \\
\hline
\end{tabular}

peut être produite, probablement, au cours de la consommation ou la vente progressives. Car la cuisson du caillé, la conservation prolongée, la concentration en sel, etc. sont, probablement, capables de détruire les contaminations initiales en bactéries coliformes.

La flore totale des échantillons a des valeurs peu importantes comprises entre 2000 et 20000 germes par gramme de fromage. Ceci est dû, sans doute, à la cuisson du caillé et la longue conservation du produit. 


\section{Rés u mé}

Le « Fromage en outre » est une des plus importantes spécialités fromagères préparées dans les régions de Tavalèche et Assalème de l'Iran.

L'affinage et la conservation de ce fromage se réalisent dans des outres fabriquées à partir de la peau des moutons et des chèvres, d'où son nom. La technologie traditionnelle de ce fromage est transmise de génération en génération depuis des siècles.

La détermination de l'azote soluble montre qu'il s'agit d'un fromage affiné.

La concentration élevée en sel de ce fromage a, sans doute, une influence certaine sur l'évolution de la flore microbienne et les qualités organoleptiques du produit.

\section{S u $\mathrm{m} \mathbf{m}$ a r y}

"Khikki-cheese » is one of the major type of cheeses produced in Tavalesh and Assalem located in Gillan district of Iran. The nam is refered to a sack made of a whole sheep or goat, which is used as container for storing the cheese. The method for producing this type of cheese has passed through years from generation to generation and until now.

Determination of soluble protein in this cheese proved, that it is a ripend cheese.

The salt content of the cheese at relativly high concentration is playing an important role on its microbial evolution and organoleptic quality.

Reçu pour publication en avril 1975.

\section{Références}

[1] Lamé (H.) et Hexmati (M.) (1973). - Contribution à l'étude du " Fromage en pot " de l'Iran. Le Lait, LIII, 163-168.

[2] HoRwitz (W.), Editor (1965). - AOAC, Methods of analysis. 10 th edition, Published by the AOAC Washington (U.S.A.).

[3] Kosikowski (F.) (1966). - Cheese and Fermented Milk Foods. Published by the Author, 2nd edition, New-York (U.S.A.).

[4] Walter (W. G.), Editor (1967). - Standard Methods. 12th edition, Published by American Public Health Assoc. Inc., New-York (U.S.A.).

[5] Newlander (J. A.) and Atherton (H. V.) (1964). - The Chemistry and Testing of Dairy Products. Olsen, 3rd edition, Wisconsin (U.S.A.). 